\title{
Ist das Öffnen eines Notenständers trivial?
}

\author{
Denis Jordan, Marcel Steiner
}

\begin{abstract}
Denis Jordan wurde 1967 in Bern geboren und ist französischsprachig aufgewachsen. Als Elektroingenieur entwickelte er eine Simulationssoftware, welche im Bereiche der Sonnenenergienutzung eingesetzt wird. Anschliessend studierte er an der Universität Bern Mathematik und Physik. Neben seiner Lehrtätigkeit am Gymnasium Burgdorf befasst er sich mit Gelenkmechanismen und Wavelets. Viel Freude bereiten ihm das Flötespielen und das Musikhören, besonders bei Werken von J. S. Bach. Er unternimmt zudem gerne Ausflüge in die Berge.

Marcel Steiner, geboren 1972 im Berner Oberland, studierte Mathematik und Physik an der Universität Bern. Zur Zeit arbeitet er als Assistant-Doctorant am Département de Mathématiques der EPF Lausanne, wo er sich im Rahmen seiner Dissertation mit Riemannscher Geometrie und Gelenkmechanismen beschäftigt. Er ist ein überzeugter Fahrradfahrer und liebt ausgedehnte Bergwanderungen. Klassische Musik und die italienische Sprache haben es ihm besonders angetan.
\end{abstract}

\section{Einleitung}

Haben Sie sich beim Öffnen eines Notenständers auch schon gewundert, als dessen Gestänge mit spontaner Biegung reagierte? Zugegeben, dies ist eine etwas beklemmende Frage. Nach einer derartigen mechanischen Beanspruchung des filigranen Notenständers ist es durchaus möglich, dass er kaum mehr als solcher Verwendung findet. Dass das sachgemässe Öffnen eines Notenständers aber keine Frage des Zufalls bleiben muss,

Über Dampfmaschinen, Lokomotiven und Flugsimulatoren bis hin zur Robotik dürfte den meisten Leserinnen und Lesern der Begriff des Gelenkmechanismus als Anordnung von Stangen, die durch Gelenke verbunden sind, bestens bekannt sein. Für die Anwendung von besonderer Bedeutung ist natürlich der Raum der möglichen Stellungen eines Gelenkmechanismus. Diese Menge wird im folgenden als Realisierungsraum des Gelenkmechanismus bezeichnet. Es ist überraschend, dass das Studium der Gelenkmechanismen mit der nicht unmittelbar anwendungsorientierten algebraischen Geometrie, d.h. mit dem Studium von Nullstellengebilden polynomialer Funktionen, in engster Verbindung steht. Wie im folgenden eindrücklich illustriert wird, ist nämlich der Realisierungsraum eines Gelenkmechanismus eine reelle algebraische Varietät. Ebenso diskutieren die Autoren die Umkehrung dieser Fragestellung. $j k$ 
möchten wir Ihnen nachfolgend etwas näherbringen. Um das Phänomen zu untersuchen, betrachten wir den Realisierungsraum des Notenständers, welcher als spezieller Gelenkmechanismus in der Ebene aufgefasst werden kann.

\section{Realisierungsräume von Gelenkmechanismen}

Ein Gelenkmechanismus lässt sich, einfach ausgedrückt, als eine endliche Anordnung von Stangen festgelegter Längen auffassen, welche durch Gelenke verbunden sein dürfen. Nachfolgend seien diese stets in der Ebene oder im Raum eingebettet. Für sie soll ein Buchstabe der Form $\mathscr{G}$ reserviert sein. Unter Berücksichtigung dieser Voraussetzungen lassen sich Gelenkmechanismen in vielen Fällen bewegen. Gleich hier möchten wir in qualitativer Weise den Realisierungsraum eines Gelenkmechanismus kennenlernen: Der (topologische) Raum aller zulässigen und mit einer Orientierung versehenen geometrischen Formen eines Gelenkmechanismus, heisst Realisierungsraum. Dieser kann ebenso als Raum aller Realisierungen modulo orientierungserhaltender Isometrien eingeführt werden.

Realisierungsräume von Mechanismen sind seit Beginn der Industrialisierung Gegenstand kinematischer Betrachtungen: Für die Entwicklung der Dampfmaschine durch den englischen Ingenieur James Watt (1736-1819) war die Kenntnis aller möglichen Stellungen der Gestänge zwischen Kolben und Antriebsachse von existentieller Bedeutung. Es war unter anderem die Frage zu beantworten, ob bei einem Kolbenhub für jedes Gelenk und jede Stange eine eindeutige Bewegung resultiert.

Heute ist eine umfassende Betrachtung solcher Mechanismen auch für die Robotik von Interesse, vgl. den Übersichtsartikel [1]. Ein Paradebeispiel hierfür ist die StewartPlattform. Wer hat nicht schon von Flugsimulatoren gehört, wie sie zur Pilotenausbildung eingesetzt werden? Es handelt sich um ein auf beweglichen Stelzen befestigtes Cockpit, dessen Position nicht nur die Fluglage des Flugzeuges angibt, sondern durch Bewegungen auch die Beschleunigungen bis zu einem gewissen Grad simulieren kann. Insbesondere haben wir es aber mit einem Gelenkmechanismus zu tun, dessen Stangen im Raum eingebettet sind. Zur korrekten Funktionsweise der Apparatur stellt sich folgende (schwierige) mathematische Frage: Impliziert eine gegebene Position des Cockpits eine eindeutige Stellung der Stangen, und falls nicht, wie stark wird diese Eindeutigkeit verletzt?

Zum Teil inspiriert durch die technischen Anwendungen befassen sich seit geraumer Zeit ebenso Mathematiker mit Gelenkmechanismen und ihren Realisierungsräumen. Und natürlich haben diese Betrachtungen auch umgekehrt obengenannte Entwicklungen der Technik stimuliert. Die Entdeckung eines Gelenkmechanismus, der mit einem seiner Gelenke einen Geradenabschnitt in der Ebene zeichnen kann, konnte für verschiedene Anwendungen von Bedeutung sein. Dessen Existenz war aber bis Mitte des neunzehnten Jahrhunderts umstritten. Es war schliesslich General Charles-Nicolas Peaucellier, der 1864 erstmals einen solchen Gelenkmechanismus postulierte.

Der berühmte französische Mathematiker Henri Lebesgue ${ }^{1)}$ schreibt hierzu: "Le guidage rectiligne d'un point a d'abord été réalisé approximativement. ... La possibilité du

1) vgl. [9], 83-84 


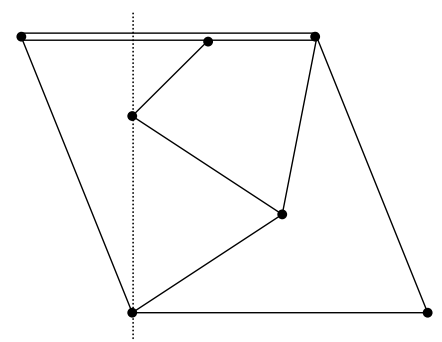

Fig. 1 Eine mögliche Anordnung eines Gelenkmechanismus, der eine Gerade zeichnet.

guidage rectiligne exact d'un point par système articulé a été mise en doute au milieu $d u \mathrm{XIX}^{e}$ siècle; comme les appareils ne permettent d'atteindre que des points à distance finie, par des mouvements de révolution ou d'oscillation, il n'était pas invraisemblable qu'on ne puisse tracer que des ovales ou des lacets plus ou moins aplatis. ..."

Etwas später konnte Alfred Kempe ein Universalitätstheorem in folgendem Sinne beweisen ${ }^{2)}$ : "Toute courbe algébrique peut être tracée à l'aide d'un système articulé."

Was aber ist eine algebraische Kurve? Zunächst ist eine algebraische Varietät $V$ nichts anderes als die Nullstellenmenge von Polynomen. Für ein reelles Polynom $f\left(x_{1}, \ldots, x_{n}\right)$ in den Variablen $x_{1}, \ldots, x_{n}$ lässt sie sich wie folgt schreiben:

$$
V=\left\{\left(x_{1}, \ldots, x_{n}\right) \in \mathbb{R}^{n} \mid f\left(x_{1}, \ldots, x_{n}\right)=0\right\} .
$$

Es handelt sich dann um eine reelle algebraische Varietät. Eine algebraische Kurve ist nun eine eindimensionale algebraische Varietät.

Es ist heute wohlbekannt, dass der Realisierungsraum eines ebenen Gelenkmechanismus immer eine algebraische Varietät ist. Die umgekehrte Frage ist aber schwieriger zu beantworten: Unter welchen Umständen ist eine algebraische Varietät der Realisierungsraum eines geeigneten Gelenkmechanismus? In dieser Form ist sie bis heute nicht beantwortet. Die Autoren konnten kürzlich zeigen, dass eine etwas abgeschwächte Form richtig ist:

Universalitätstheorem. Für jede kompakte, reelle algebraische Varietät $V$ gibt es ei-

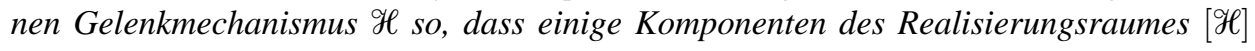
homöomorph zu $\mathrm{V}$ sind.

Der Beweis des Universalitätstheorems, vgl. [6], liefert uns einen Algorithmus zur expliziten Konstruktion des jeweiligen Gelenkmechanismus. Leider wird letzterer derart unübersichtlich, dass das Theorem als reine Existenzaussage aufzufassen ist. Es stellt sich die Frage nach einfachen Gelenkmechanismen, um beispielsweise alle Flächen zu realisieren. In [7] ist folgendes Ergebnis zu finden:

Theorem 2.1. Für jede kompakte, geschlossene, orientierbare und zusammenhängende Fläche $\Sigma_{g}$ mit beliebigem Geschlecht $g \in \mathbb{N}$ gibt es einen "einfachen" Gelenkmechanismus $\mathscr{Y}_{g}$ derart, vgl. Figur 2, dass $\left[\mathscr{Y}_{g}\right]$ homöomorph zu $\Sigma_{g}$ ist.

2) vgl. [9], 84 


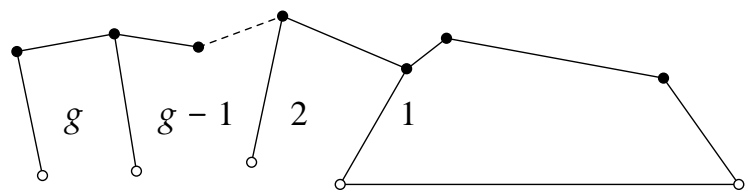

Fig. 2 Der Gelenkmechanismus $\mathscr{Y}_{g}$

Weitere Beispiele für überblickbare Gelenkmechanismen sind m-Polygone: Es sind dies spezielle Gelenkmechanismen bestehend aus $m$ Gelenken, die zyklisch mit $m$ Stangen verbunden sind, zusammen also eine geschlossene "Kette" bilden.

Denkbar einfach ist das in der Ebene liegende 3-Polygon $\mathscr{P}_{3}$. Ein Dreieck $\mathscr{P}_{3}$ lässt sich niemals deformieren, dies erlaubt uns, den Realisierungsraum ohne Schwierigkeiten anzugeben: $\left[\mathscr{P}_{3}\right]$ besteht aus einem Punkt, falls die Summe der Längen zweier Stangen mit der Länge der dritten übereinstimmt, andernfalls aus zwei disjunkten Punkten. Im letzten Fall ist die eine Realisierung von $\mathscr{P}_{3}$ das Spiegelbild der anderen, und die beiden lassen sich in der Ebene nicht ineinander überführen.

Tip. Ein einfaches Modell aus Trinkhalmen ist oft eine nützliche Hilfe, um die Funktionsweise eines Gelenkmechanismus in erster Näherung zu verstehen.

Sollten wir nun mehr Interesse auf polygonale Gelenkmechanismen und ihre Realisierungsräume geweckt haben, so raten wir der interessierten Leserin und dem interessierten Leser, 4- und 5-Polygone in der Ebene zu studieren. Für alle solchen lassen sich die Realisierungsräume explizit angeben. J.-C. Hausmann berechnete Realisierungsräume aller ebenen 4-Polygone, vgl. [2]:

Theorem 2.2. Es sei $\mathscr{P}_{4}$ ein 4-Polygon in der Ebene. Ist der Realisierungsraum eine Mannigfaltigkeit, dann ist $\left[\mathscr{P}_{4}\right]$ homöomorph zu $S^{1}$ oder $S^{1} \amalg S^{1}$, wobei $S^{1}$ die eindimensionale Sphäre ist. Andernfalls besitzt der Realisierungsraum Singularitäten, die lokal (eindimensionale) Doppelkegel sind.

Mit Hilfe der Morse-Theorie konnte T.F. Havel zeigen, vgl. [4], dass der Realisierungsraum eines Pentagons, das ist ein 5-Polygon mit gleichen Stangen, eine Fläche vom Geschlecht vier ist. Später untersuchte B. Jaggi in seiner Dissertation, vgl. [5], beliebige $m$-Polygone in der Ebene. Dabei gelang es ihm, alle 5-Polygone nach ihren Realisierungsräumen zu klassifizieren. Für einen etwas anderen Zugang sei auch [3] erwähnt.

Theorem 2.3. Es sei $\mathscr{P}_{5}$ ein 5-Polygon in der Ebene. Ist der Realisierungsraum eine Mannigfaltigkeit, dann ist $\left[\mathscr{P}_{5}\right]$ homöomorph zu $S^{2}, \Sigma_{1} \amalg \Sigma_{1}$ oder $\Sigma_{g}$ für $g \in\{1, \ldots, 4\}$, wobei $S^{2}$ die zweidimensionale Sphäre und $\Sigma_{g}$ eine Fläche vom Geschlecht $g$ ist. Andernfalls besitzt der Realisierungsraum Singularitäten, die lokal Doppelkegel sind.

Einfache Beweise der beiden Theoreme 2.2 und 2.3 finden sich in der zusammenfassenden Arbeit von M. Kapovich und J. Millson, vgl. [8].

Dank. Unser spezieller Dank gilt J.-C. Hausmann und P. Mani, die uns Gelenkmechanismen und deren Realisierungsräume schmackhaft machten. 


\section{Der Notenständer - ein spezieller Gelenkmechanismus}

\subsection{Die Grundbegriffe}

Zuerst ersetzen wir die intuitive Vorstellung eines Gelenkmechanismus durch eine exakte mathematische Definition:

Definition 3.1. Das Tripel $\mathscr{G}=(E, K, d)$ bestehend aus

(1) einer Eckenmenge $E=\left\{E_{1}, \ldots, E_{m}\right\}$ und

(2) einer Kantenmenge $K=\left\{\left\{E_{i_{1}}, E_{j_{1}}\right\}, \ldots,\left\{E_{i_{k}}, E_{j_{k}}\right\}\right\}$ mit $i_{l}, j_{l} \in\{1, \ldots, m\}, i_{l} \neq j_{l}$ und

(3) einer Kantenbewertung $d: K \rightarrow \mathbb{R}_{+}$, welche jeder Kante $\left\{E_{i_{l}}, E_{j_{l}}\right\}$ in $K$ eine Länge (Gewicht) $d\left(E_{i_{l}}, E_{j_{l}}\right) \in \mathbb{R}_{+}$zuordnet,

heisst gewichteter Graph.

Besteht ein solcher aus mehreren Komponenten, so lassen sich diese stets einzeln betrachten, weshalb nachfolgend o.E.d.A. immer von zusammenhängenden gewichteten Graphen die Rede ist.

Wir nennen $\mathscr{G}=(E, K, d)$ realisierbar in $\mathbb{R}^{n}$, falls es eine Figur $\xi: E \rightarrow \mathbb{R}^{n}$ so gibt, dass $\left|\xi\left(E_{i}\right)-\xi\left(E_{j}\right)\right|=d\left(E_{i}, E_{j}\right)$ für alle Kanten $\left\{E_{i}, E_{j}\right\}$ in $K$ gilt. Eine Realisierung eines gewichteten Graphen $\mathscr{G}=(E, K, d)$ in $\mathbb{R}^{n}$ ist ein $m$-Tupel $\xi=\left(x_{1}, \ldots, x_{m}\right) \in\left(\mathbb{R}^{n}\right)^{m}$ der Punkte $x_{j}=\xi\left(E_{j}\right)$ in $\mathbb{R}^{n}$.

Beachte: nicht jeder gewichtete Graph lässt sich in einem gegebenen Einbettungsraum realisieren: Ein zyklischer Graph mit Kantenlängen 1,2 und 4 erlaubt überhaupt keine Realisierung, weil eine der drei Dreiecksungleichungen verletzt ist. Ein reguläres Tetraeder mit Kantenlänge 1 lässt sich zwar in $\mathbb{R}^{3}$ realisieren, nicht aber in $\mathbb{R}^{2}$. In der Arbeit [10] findet sich eine Antwort zur Fragestellung, wann ein gegebener gewichteter Graph in $\mathbb{R}^{n}$ realisierbar ist. Fortan betrachten wir nur zusammenhängende gewichtete Graphen, die in $\mathbb{R}^{n}$ realisierbar sind, und bezeichnen sie einfach als Gelenkmechanismen.

Der Raum

$$
[\mathscr{G}]=\left\{\xi \text { Realisierung von } \mathscr{G} \text { in } \mathbb{R}^{n}\right\} / \mathrm{Iso}^{+}\left(\mathbb{R}^{n}\right)
$$

aller möglichen Realisierungen eines Gelenkmechanismus $\varphi=(E, K, d)$ in $\mathbb{R}^{n}$ wird als Realisierungsraum von $\mathscr{G}$ in $\mathbb{R}^{n}$ bezeichnet, wobei $\operatorname{Iso}^{+}\left(\mathbb{R}^{n}\right)$ die orientierungserhaltende Isometriegruppe von $\mathbb{R}^{n}$ ist. Massgebend sind also nur die möglichen orientierten Formen eines Gelenkmechanismus und nicht deren Position im Einbettungsraum $\mathbb{R}^{n}$.

Interessant ist der Begriff des Realisierungsraumes [G] vor allem dann, wenn sich dieser für einen gegebenen Gelenkmechanismus $\mathscr{G}$ auch explizit berechnen lässt. Eine wesentliche Vereinfachung erhalten wir für ebene Gelenkmechanismen (d.h. solche in $\mathbb{R}^{2}$ ), wenn eine beliebige Kante von $\mathscr{G}$ in willkürlich gewählter Lage festgehalten wird: Die Quotientenbildung in der Definition von [G] dient nämlich nur dazu, Realisierungen von $\mathscr{G}$, die sich durch Translationen bzw. Rotationen unterscheiden, nicht mehrfach zu berücksichtigen. Definiere den Arbeitsraum

$$
\mathbb{A}\left(E_{j}\right)=\left\{\xi\left(E_{j}\right) \mid \xi \in[G]\right\} \subset \mathbb{R}^{2}
$$


der Ecke $E_{j}$ als Menge aller möglichen Punkte $\xi\left(E_{j}\right) \in \mathbb{R}^{2}$. Der Realisierungsraum [G] lässt sich mit dem Arbeitsraum einer Ecke in Zusammenhang bringen, indem alle Realisierungen von $\xi \in[G]$ angegeben werden, die für einen Punkt $\xi\left(E_{j}\right) \in \mathbb{A}\left(E_{j}\right)$ möglich sind: Wir definieren dazu die Projektion

$$
\begin{aligned}
\pi:[G] & \longrightarrow \mathbb{A}\left(E_{j}\right) \\
\xi & \longmapsto \xi\left(E_{j}\right)
\end{aligned}
$$

und erhalten eine verzweigte Faserung ${ }^{3)}$ über $\mathbb{A}\left(E_{j}\right)$, wobei $\left\{\xi \in[G] \mid \xi\left(E_{j}\right)=p\right\}=$ $\pi^{-1}(p)$ die Faser über $p \in \mathbb{A}\left(E_{j}\right)$ ist. Natürlich gilt dann insbesondere $\pi^{-1}\left(\mathbb{A}\left(E_{j}\right)\right)=[G]$.

\subsection{Der Notenständer}

Nach diesen allgemeinen Definitionen möchten wir uns wieder einem Gelenkmechanismus widmen, dem Notenständer, und seinen Realisierungsraum explizit bestimmen. Letzterer wird uns ebenso über die korrekte Handhabung wie über die Gründe einer Beschädigung infolge unsachgemässer Bedienung Auskunft geben können. Falls Sie, verehrte Leserin und verehrter Leser, zu diesem Zeitpunkt bereits den Eindruck nicht loswerden, die Mathematik des vorigen Abschnittes habe Sie mehr gefordert, als jemals das Öffnen des genannten Gegenstandes, sollten Sie keineswegs kapitulieren. Mit nachfolgenden Überlegungen lassen sich die eingeführten Begriffe anwenden und festigen. Im folgenden Abschnitt findet sich eine Bedienungsanleitung für den Notenständer.

Es sei hier vermerkt, dass im Sinne obiger Definitionen ausschliesslich die Notenablagefläche als ebener Gelenkmechanismus aufzufassen ist und nicht etwa das allenfalls vorhandene Teleskopbein. Sie besteht bekanntlich aus zwei symmetrischen Teilen, die einzeln aufgeklappt und je als einzelne Gelenkmechanismen betrachtet werden dürfen. Ohne Einschränkung der Allgemeinheit betrachten wir den rechten Flügel bestehend aus fünf Ecken $A, B, C, D, E$ in der Ebene, vier Kanten $\{A, B\},\{B, C\},\{C, D\},\{D, A\}$ der Länge 1 sowie zwei Kanten $\{B, E\},\{E, D\}$ der Länge $\sqrt{2} / 2$, vgl. Figur 3 . Er sei mit $\mathcal{N}$ bezeichnet.
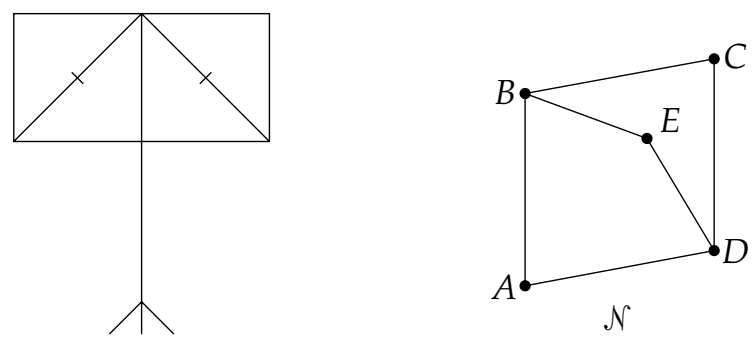

Fig. 3 Notenständer und Gelenkmechanismus $\mathcal{N}$

3) Es handelt sich nicht um eine verzweigte Überlagerung, weil im allgemeinen $\pi^{-1}(p)$ nicht diskret ist, und auch nicht um eine Faserung, weil im allgemeinen die lokale Trivialität verletzt ist. Hingegen ist die Figur $\pi$ stets surjektiv. 
Es seien von nun an $\xi(A)=(0,0)$ und $\xi(B)=(0,1)$, dies legt die Position der Kante $\{A, B\}$ fest. Dann ist

$$
\mathbb{A}(D)=\left\{(\sin \varphi, \cos \varphi) \mid \varphi \in\left[\frac{-\pi}{2}, \frac{\pi}{2}\right]\right\}
$$

der Arbeitsraum der Ecke $D$. Für jeden Punkt $p$ in $\mathbb{A}(D)$ betrachten wir die möglichen Realisierungen, welche uns schliesslich zur verzweigten Faserung $\pi:[\mathcal{N}] \longrightarrow \mathbb{A}(D)$ führen.

Ist $p=(1,0)$, dann sind genau zwei Realisierungen von $\mathcal{N}$ zulässig, welche wir mit $\xi_{12}$ und $\xi_{34}$ bezeichnen. Für $p=(-1,0)$ sind es ihre spiegelbildlichen Realisierungen $\xi_{12}^{\prime}$ und $\xi_{34}^{\prime}$. Bei beliebigem $p \in\{(\sin \varphi, \cos \varphi) \mid \varphi \in] 0, \frac{\pi}{2}[\} \subset \mathbb{A}(D)$ erhalten wir je vier Realisierungen $\xi_{1 \varphi}, \xi_{2 \varphi}, \xi_{3 \varphi}$ und $\xi_{4 \varphi}$ und entsprechend ihre Spiegelbilder $\xi_{1 \varphi}^{\prime}, \xi_{2 \varphi}^{\prime}, \xi_{3 \varphi}^{\prime}$ und $\xi_{4 \varphi}^{\prime}$ für jedes $p \in\{(\sin \varphi, \cos \varphi) \mid \varphi \in]-\frac{\pi}{2}, 0[\}$.

Schliesslich ist die Faser $\pi^{-1}(p)$ dann nicht diskret, wenn $p=(0,1)$ ist: $\{\xi(C) \mid \xi(D)=$ $p\}$ und $\{\xi(E) \mid \xi(D)=p\}$ bilden zwei individuelle Kreise wie Figur 4(b) veranschaulicht. Insgesamt erhalten wir die verzweigte Faserung

$$
\pi^{-1}(p)=\left\{\begin{array}{cl}
p t \amalg p t & \text { falls } p \in\{(-1,0),(1,0)\}, \\
p t \amalg p t \amalg p t \amalg p t & \text { falls } p \in\{(\sin \varphi, \cos \varphi) \mid \varphi \in]-\frac{\pi}{2}, 0[\cup] 0, \frac{\pi}{2}[\}, \\
S^{1} \times S^{1} & \text { falls } p=(0,1),
\end{array}\right.
$$

wie sie auch in Figur 4 dargestellt ist.

(a)

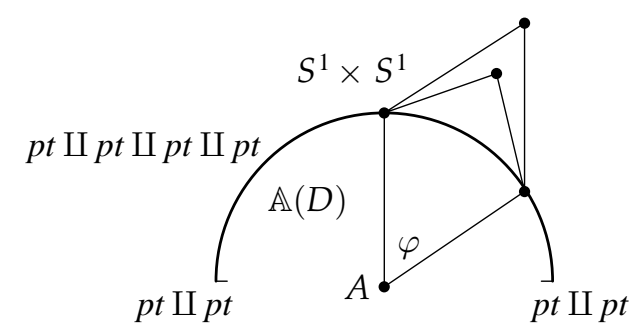

(b)

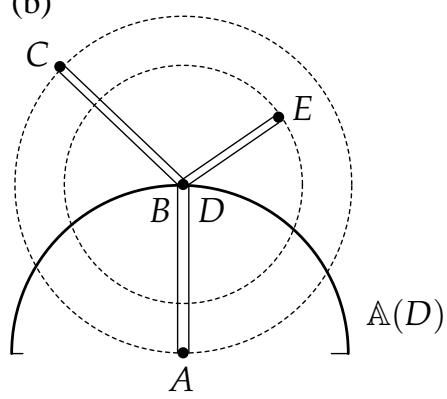

Fig. 4 Der Arbeitsraum $\mathbb{A}(D)$ mit Faserung des abstrahierten Notenständers

Aus diesen Informationen bestimmen wir nun den Realisierungsraum: Im Torus $S^{1} \times S^{1}=$ $\pi^{-1}(p)$ für $p=\xi(D)=(0,1)$ beginnen wir mit den vier degenerierten Realisierungen. Das sind diejenigen, für welche die Gelenke $A, B, D, C$ und $E$ des Notenständers auf einer Geraden liegen. Wir bezeichnen sie mit $\xi_{10}, \xi_{20}, \xi_{30}$ und $\xi_{40}$.

Wie die Sequenz (1) in Figur 5 zeigt, wird ausgehend von $\xi_{10}$ der erwünschte geöffnete Zustand des Notenständers, also die Realisierung $\xi_{12}$ erreicht, indem alle Realisierungen 
(1)

(2)

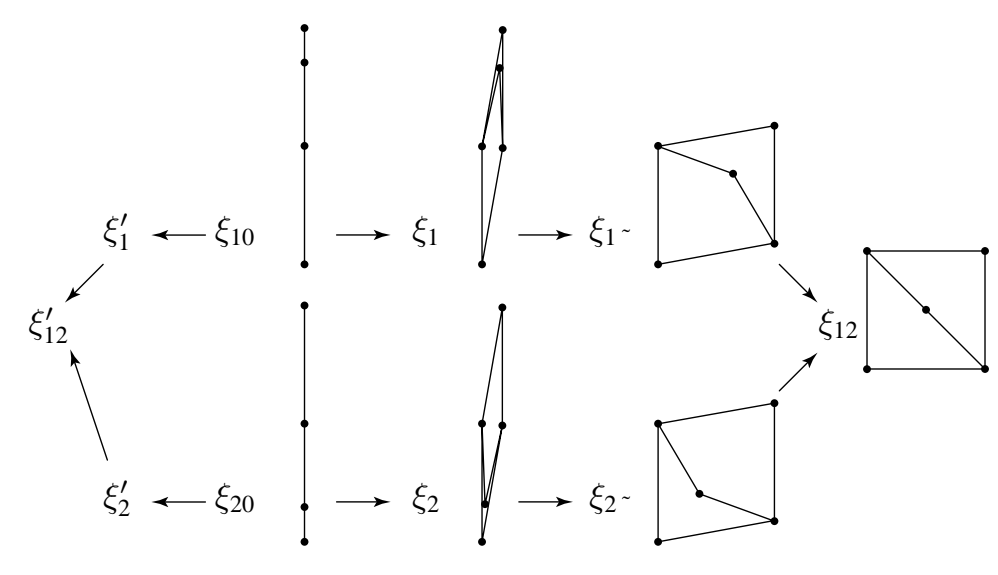

(3)

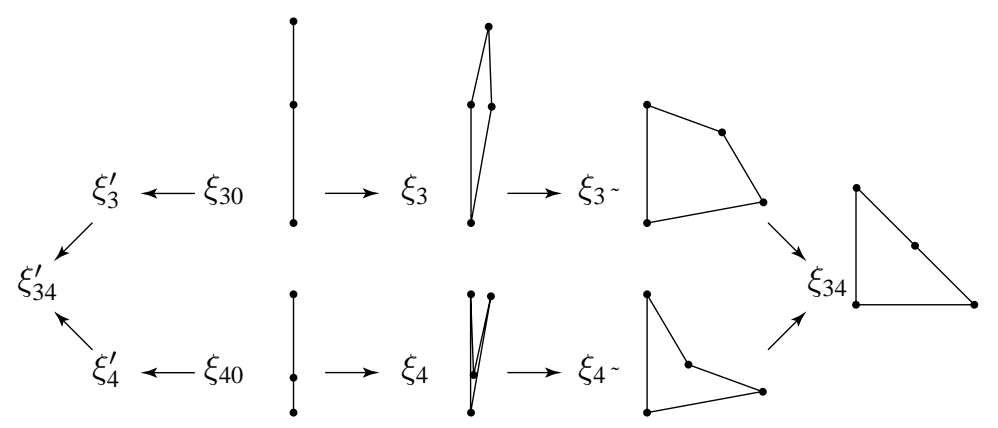

Fig. 5 Vier verschiedene Öffnungssequenzen, von denen (1) und (2) zur gewünschten offenen Realisierung führen.

$\xi_{1 \varphi}$ für $\left.\varphi \in\right] 0, \frac{\pi}{2}[$ mit einer nach rechts orientierten Öffnung durchlaufen werden. Ebenso ist es denkbar, diesen ausgehend von $\xi_{20}$ über die Realisierungen $\xi_{2 \varphi}$ für alle $\left.\varphi \in\right] 0, \frac{\pi}{2}[$ der Sequenz (2) zu erreichen.

Analoges gilt für eine Öffnung aus den entarteten Realisierungen $\xi_{30}$ bzw. $\xi_{40}$. Hier wird aber nicht der für einen Notenständer übliche Zustand erreicht, sondern die Realisierung $\xi_{34}$, wie in den Sequenzen (3) und (4) illustriert.

Die spiegelbildlichen Realisierungen $\xi_{12}^{\prime}$ bzw. $\xi_{12}^{\prime}$ des geöffneten Notenständers werden ausgehend von $\xi_{10}$ und $\xi_{20}$ bzw. $\xi_{30}$ und $\xi_{40}$ mit einer Öffnung nach links erreicht. Hier ist $\varphi \in]-\frac{\pi}{2}, 0[$.

Anhand der verzweigten Faserung über $\mathbb{A}(D)$ und obiger Öffnungssequenzen lässt sich nun die Bestimmung des Realisierungsraumes $[\mathcal{N}]$ von $\mathcal{N}$ vervollständigen. Dieser besteht aus einem Torus, vier Punkten und acht offenen Intervallen. Je zwei der Intervalle werden an einem Ende mit einem der Punkte verklebt und verschmelzen so insgesamt zur halben Anzahl. An ihren Enden werden sie paarweise in den Punkten $\xi_{10}, \xi_{20}$, respektive $\xi_{30}, \xi_{40}$ des Torus angeklebt. Die Öffnungssequenzen in Figur 5 verraten die Stellen, an welchen die Verklebungen am Torus stattfinden, und es folgt der Realisierungsraum $[\mathcal{N}]$, wie er in Figur 6 dargestellt ist. 


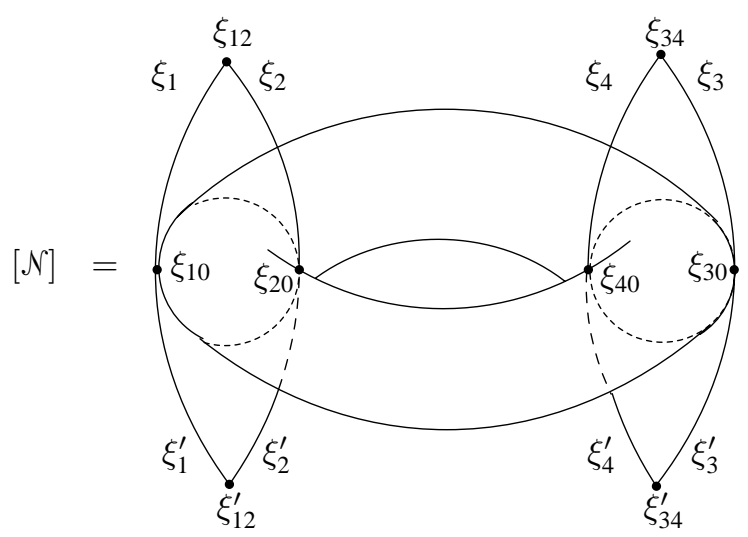

Fig. 6 Der Realisierungsraum von $\mathcal{N}$

\subsection{Praktische Folgerungen}

Die zum Öffnen des Notenständers erforderlichen Grundlagen sind nun bereitgestellt. Wir haben dabei entdeckt, dass die Betrachtung des rechten Flügels genügt, um unser Grundproblem zu lösen. Ausgehend von einer Realisierung im Torus, wie in Figur 4(b) illustriert, sind es also genau die durch $\xi_{10}$ oder $\xi_{20}$ führenden stetigen Wege, die das Öffnen des Flügels gestatten, das heisst in $\xi_{12}$ enden. Die Gelenke $C$ und $E$ müssen dazu auf einer Geraden durch $A$ und $B$ angeordnet sein, wobei das Gelenk $C$ nicht auf $A$ liegen darf, siehe $\xi_{10}, \xi_{20}$ in Figur 5. Erst dann lässt sich der Flügel erfolgreich auf einem der eindimensionalen Bereiche des Realisierungsraumes aufklappen.

Die zu $\xi_{12}$ spiegelbildliche Realisierung $\xi_{12}^{\prime}$ ist für die Praxis nicht von Bedeutung, weil sich der Notenständer durch konstruktive Massnahmen nicht genau wie ein ebener Gelenkmechanismus verhält und eine derartige Öffnung nicht zulässt. Natürlich verhält sich der linke Flügel des Notenständers wie der rechte.

Es ist nun einsichtig: Der Laie wird den Notenständer mit grosser Wahrscheinlichkeit nicht ohne Zwischenfälle öffnen können. Vielmehr dreht er gleichzeitig die Gelenke $E$ und $C$, ohne zu wissen, dass er damit ziellos Realisierungen auf dem zweidimensionalen Torus erzeugt. Seine Versuche, die Stangen auseinander zu biegen, dürften meist misslingen. Es wäre reiner Zufall, wenn er mit seiner Methode eine der notwendigen Realisierungen $\xi_{10}$ oder $\xi_{20}$ trifft, um eine stetige Kurve auf $\mathcal{N}$ nach $\xi_{12}$ einzuleiten.

Nicht so der erfahrene Musiker: Er nutzt die ideale Öffnungsstrategie, indem er den geschlossenen Notenständer $\xi_{40}$ auf kürzestem Weg durch eine Drehung des Gelenkes $C$ nach $\xi_{20}$ überführt, um anschliessend seine Partitur auf dem sich einfach öffenden Notenständer $\xi_{12}$ auflegen zu können - gewusst wie.

\section{Literatur}

[1] D.R. Baker, Some Topological Problems in Robotics, Math. Intelligencer 12 no. 1, 1990, 66-76.

[2] J.-C. Hausmann, Sur la topologie des bras articulés, Springer Lecture Notes in Mathematics 1474, 1989, $146-159$.

[3] J.-C. Hausmann und A. Knutson, Polygon Spaces and Grassmannians, Enseign. Math. (2) 43, 1997, 173-198. 
[4] T.F. Havel, The Use of the Distance Function as Coordinates in Computer-Aided Proofs of Theorems In Euclidean Geometry, J. Symbolic Comput. 11, 1991, 579-593.

[5] B. Jaggi, Punktmengen mit vorgeschriebenen Distanzen und ihre Konfigurationsräume, Inauguraldissertation, Universität Bern, 1992.

[6] D. Jordan und M. Steiner, Configuration Spaces of Mechanical Linkages, Discrete Comput. Geom. 22, 1999, 297-315.

[7] D. Jordan und M. Steiner, Compact Surfaces as Configuration Spaces of Mechanical Linkages, erscheint in Israel J. of Math.

[8] M. Kapovich und J. Millson, On the Moduli Space of Polygons in the Euclidean Plane, J. of Differential Geom. 42, 1995, 133-164.

[9] H. Lebesgue, Leçons sur les constructions géométriques, Gaulthier-Villars, Paris, 1949, (Nachdruck 1987).

[10] O. Mermoud und M. Steiner, Configuration Spaces of Weighted Graphs Imbedded in High Dimensional Euclidean Spaces, Preprint 1999.

Denis Jordan

Mathematisches Institut

der Universität Bern

CH-3012 Bern

e-mail:

denis.jordan@math-stat.unibe.ch

\author{
Marcel Steiner \\ Département de Mathématiques \\ EPF Lausanne \\ CH-1015 Lausanne \\ e-mail: \\ marcel.steiner@epfl.ch
}

